\title{
Effects of music playing on biological molecules
}

\author{
Catia Algieri ${ }^{1}$, Claudio Guarnaccia*2, Vincenzo Barone ${ }^{3}$, Maria Raffaella Gullo ${ }^{1}$, and Laura Donato*1 \\ ${ }^{1}$ Research Institute on Membrane Technology, ITM-CNR, University of Calabria, Via P. Bucci, Cubo 17/C, 87030 Rende (CS), Italy \\ ${ }^{2}$ Department of Civil Engineering, University of Salerno, Via Giovanni Paolo II 132, Fisciano (SA), Italy \\ ${ }^{3}$ DINCI Department University of Calabria, 87030 Rende (CS), Italy
}

\begin{abstract}
In this work the effect of two different musical pieces (called "Reminiscenza" and "Pioggia") on the tyrosinase enzyme activity was investigated. The 1-DOPA production by the enzymatic reaction was measured during the continuous playing of these musical pieces. Experiments were performed in the laboratories of the Institute of Membrane Technology (ITM) of the Italian National Council for Research (CNR). The results showed that a positive effect was exercised by the "Reminiscenza" piece, which determined an increase of the specific activity about of $30 \%$ with respect to the value measured in the absence of sound. On the contrary, "Pioggia" piece had a detrimental effect on the enzymatic process. In particular, a time lag on the 1-DOPA production, during the first minutes of the reaction, was detected. After this period, an increase of the reaction velocity occurred even if the enzyme activity was lower than the value obtained in the absence of music. These results show that the peculiar characteristics of a musical piece can exercise a positive or negative action on biological elements and open the way to further studies in this area.
\end{abstract}

\section{Introduction}

All living organisms interact with different kind of noises and sounds present in the environment, therefore, the attention of the scientists is focused on the understanding the close relationship between sound and biological systems.

Sound is a mechanical wave that travels through a medium (air, water, etc.) transferring energy from one site to another. The sound perceived by our auditory system has a frequency between $20 \mathrm{~Hz}$ and $20 \mathrm{kHz}$ and is defined audible sound. Sounds with frequency below $20 \mathrm{~Hz}$ and above $20 \mathrm{kHz}$ are defined infrasound and ultrasound, respectively.

In literature, different studies on the effect of ultrasound on various biotechnological processes were performed. Some example are the cellular destruction for promoting the release of intracellular enzymes and organelles [1-3], and several transesterification and hydrolytic reactions $[4,5]$. Other applications are the treatment of bio sludge [6], the increase in growth rate and the size of some plants and seeds, etc. [7].

However, in this scenario it must be taken into account that very few papers deal with the effect of audible sound (in terms of frequency, sound pressure level, exposition time and source distance) on living organisms as for example plants and bacterials $[8,9]$. For example, Shaobin and co-workers [9] investigated the effect of sound waves on the cellular cycle of Escherichia coli, a bacterium of our intestine. This study evidenced that the stimulation with the audible sound $(1000,5000,10000 \mathrm{~Hz})$ resulted in an increase of the speed of cell proliferation in normal culture conditions. The lower frequency $(1000 \mathrm{~Hz})$ exercised the greatest effect. However, in osmotic stress conditions (for addition of sugars to the culture medium) the presence of the sound caused an inhibition on the cellular growth. Recently, it was also confirmed the relationship between enzyme activity and sound waves at different frequencies $(100 \mathrm{~Hz}, 2.500 \mathrm{~Hz}, 6.300 \mathrm{~Hz}, 8.000 \mathrm{~Hz}$, $10.000 \mathrm{~Hz}, 12.500 \mathrm{~Hz}$ and $16000 \mathrm{~Hz}$ ) using two types of polished rice [10].

In another paper, Chivukula and Ramaswamy studied the effect of different kind of music ( Indian Classical music, Vedic chants, Western Classical music, and Rock music) on 30 Rose (Rose chensis) plants. Authors verified that music can either stimulate or limit the plants growth, depending on the type of the played music [11]. In 2017 Ventura et al. demonstrated that stem cells are able to respond with different vibrational signatures to the sound generated by artists in the form of music or voice dialog in live performances [12].

In this work, for the first time, the effect of musical pieces (with peculiar characteristics) on the activity of the tyrosinase (polyphenol oxidase EC 1.14.18.1) during the oxidation of 1-tyrosine in 1-3,4-dihydroxyphenyl-1alanine (1-DOPA) was investigated. The l-DOPA is the preferred drug used in the treatment of Parkinson's disease $[13,14]$.

The used musical pieces, called "Reminiscenza" and "Pioggia" (by the composer G. Formigari-Bernardelli) were characterized by different frequencies, rhythm and melody. For comparison, catalytic tests in the absence of sound were also carried out.

*Corresponding author: 1.donato@itm.cnr.it , cguarnaccia@unisa.it 


\section{Materials and Methods}

\subsection{Materials}

Tyrosinase (EC 1.14.18.1 from mushroom as lyophilized powder), l-ascorbic acid (purity 99 wt.\%), l-tyrosine (purity 98 wt.\%), 1-DOPA were purchased from SigmaAldrich (Milan, Italy). Sodium phosphate monobasic (98 wt.\%) and sodium phosphate dibasic (98 wt.\%) were also supplied by Sigma-Aldrich (Milan, Italy.

The acoustic measurements were carried out with a FUSION $01 \mathrm{~dB}$ device, as described below.

\subsection{Characteristics of musical pieces}

The musical instrument used for composing the two musical pieces was a digital piano Roland HP 133 1300e.

"Reminiscenza" is characterised by a constant rhythm and melody, with a very regular repetition of the Do in $523.25 \mathrm{~Hz}$. Besides, the fundamental frequencies of the song are $523.25,587.33$ and $659.26 \mathrm{~Hz}$. Finally, it has a thetic rhythm.

The "Pioggia" piece differs from the first one being inconstant in the rhythm. In particular, the rhythm is defined in an agogic accent with a rubato, therefore, it is sometimes slow and sometimes accelerated. The fundamental frequencies are 261.63 e $293.66,523.25$ e $622.25,698.46$ and $830.61 \mathrm{~Hz}$.

\subsection{Instruments and software}

The acoustic measurements have been performed using a FUSION $01 \mathrm{~dB}$ sound level meter. This instrument is a multi-purpose tool, that can be used as a control instrument and offers evaluation, analysis and monitoring capabilities application to noise and vibration measurement in the following fields of activity, as noise exposure, musical activities, vibration of machines and vibrations of structures. It is certified Class 1 according to IEC 61672. It has been used in wireless connection, controlling by a remote tablet, in order to minimize the influence of the operator.

In order to simplify the deployment of FUSION in the field, an automatic function for the detection of a sound level calibrator is used to launch the calibration procedure without any action required from the user, other than powering up the calibrator. When FUSION detects a stable level around the predefined calibration level, it automatically starts the calibration procedure. At the end of this procedure, the instrument indicates the new calculated sensitivity and prompts the user for validation, repeat or rejection of the calibration. Information provided is stored and added up to the historical data of the instrument. In our case, the calibration has been performed before and after the measurements.
The readout and analysis software program adopted in this application is "dB Trait", produced by $01 \mathrm{~dB}$ Acoem Group. This software can be used for data analysis, since includes processing functions such as multiple indicators calculations, analysis results according to regulations as well as advanced coding capabilities, which help identify noise events and level variations.

\subsection{Evaluation of the enzyme performance}

The tyrosinase activity was assessed by following the production of 1-DOPA in a stirred thank reactor at $30{ }^{\circ} \mathrm{C}$ $[14,15]$. The reaction mixture $(100 \mathrm{~mL})$ was constituted of $50 \mathrm{mM}$ sodium phosphate buffer ( $\mathrm{pH} 7.0$ ), $2.5 \mathrm{mM}$ of 1-tyrosine and $2.5 \mathrm{mM}$ of 1 -ascorbic acid. The reaction was initiated by adding the enzymatic solution having a concentration of $3.7 \times 10-3 \mathrm{mg} \mathrm{mL}-1$. The quantitative determination of the 1-DOPA was performed on a LaChrom D7000 HPLC system (Hitachi) equipped with a UV detector. Analyses were carried out using the column Prevail C 18, $5 \mu \mathrm{m}, 250 \mathrm{~mm} \times 4.6 \mathrm{~mm}$ (Alltech, Italy). The mobile phase was $25 \mathrm{mM}$ of $\mathrm{KH}_{2} \mathrm{PO} 4(\mathrm{pH}=$ 4). The operating conditions were: flow rate of $1.0 \mathrm{~mL}$ $\min ^{-1}$ and temperature of $25^{\circ} \mathrm{C}$.

The specific activity was calculated dividing the catalytic activity for the mass of the enzyme. The catalytic activity was the linear coefficient of the straight line obtained by plotting the $\mu$ moles of the 1-DOPA produced versus time.

For evaluating the effect of sound on the enzymatic activity, reactions were carried out with and without exposure to the musical pieces. In the first case, The reaction mixture was exposed to the action of the musical piece "Reminiscenza" or "Pioggia".

Experiments were done in a controlled chamber. In particular, the reactor vessel was placed on a magnetic plate equipped with a controlled agitation system and a digital temperature control device, for maintaining constant the stirring conditions and the reaction temperature, respectively. The source of the sound was a computer (LENOVO ThinkCentre E93z) having two internal 100 integrated speakers (3 Watt). The distance between the computer and the magnetic plate was $10 \mathrm{~cm}$. During the experiments the sound pressure level was continuously measured with Fusion. Before the experiments, the correspondence between sound source percentage of volume and observed acoustic intensity level was calibrated. Pink noise has been put in output of the source and the level in the chamber has been measured, as a function of the volume percentage of the PC (PC volume).

The results are shown in Figure 1, in which a linear slope can be detected. With these results, it was possible to convert volume percentages to sound pressure levels. 


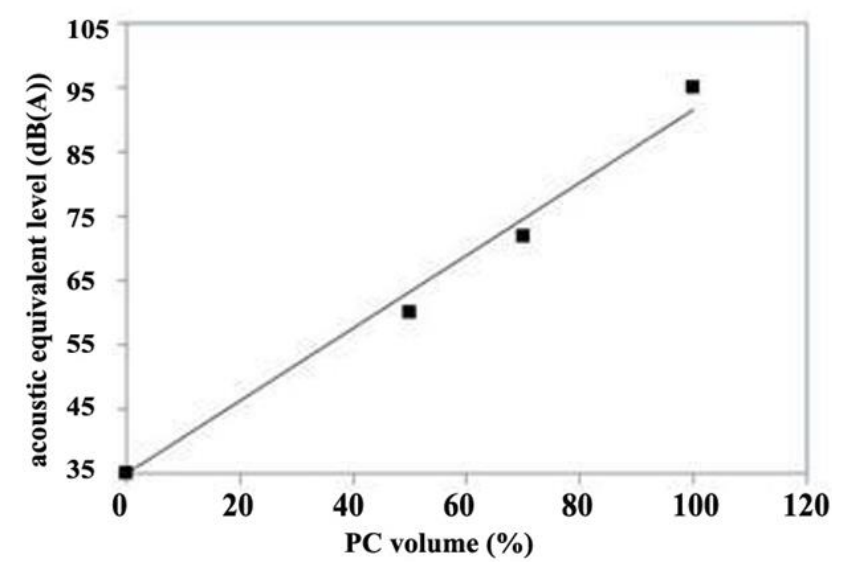

Fig. 1. Production of 1-DOPA in the time (with and without "Reminiscenza" piece).

\section{Results and discussion}

The effect of the audible sound on the tyrosinase activity for the 1-DOPA production under stimulation of "Reminiscenza" and "Pioggia" pieces was investigated. At the beginning, biocatalytic tests were performed with the "Reminiscenza", using an acoustic equivalent level of $75 \mathrm{~dB}(\mathrm{~A})$. Experimental results evidenced a positive effect of this piece on the 1-DOPA production (see Figure 1). The specific activity increased of about $30 \%$ with respect to the value measured in the absence of sound.

This behavior can be explained with the frequency resonance theory [16]: the sound frequencies provide energy to the enzyme and determine a conformational change in its structure [17]. This phenomenon makes the active sites of the enzyme more accessible for the substrate.

The experimental tests under the "stimulus" of the "Pioggia" piece were performed using the same configuration at three different PC volume: 100\%, 75\% and $50 \%$ (corresponding to an acoustic equivalent level of $81 \mathrm{~dB}(\mathrm{~A}), 75.5 \mathrm{~dB}(\mathrm{~A})$ and $60.5 \mathrm{~dB}(\mathrm{~A})$, respectively). In particular, a time lag on the 1-DOPA production was observed during the first minutes of the reaction in the presence of the musical piece. It must also be highlighted that, after the time lag, an increase of the reaction velocity was observed in all the investigated cases (see Figure 2).

However, the enzymatic activity was lower than the value obtained in the absence of music. In particular, at sound intensity of $81 \mathrm{~dB}(\mathrm{~A})$, the reduction of the 1DOPA production was of about $80 \%$ than that one observed without sound.

This behavior was less pronounced carrying out the catalytic tests at lower intensity. In this case, the reduction of the 1-DOPA amount was about $60 \%$ at an acoustic equivalent level of $75.5 \mathrm{~dB}(\mathrm{~A})$ and $50 \%$ at an acoustic equivalent level of $60.5 \mathrm{~dB}(\mathrm{~A})$.

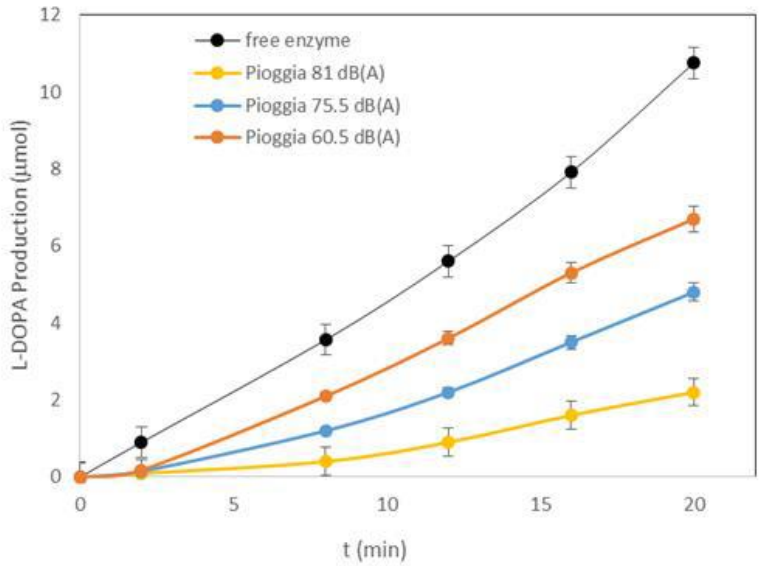

Fig. 2. Production of 1-DOPA in the time without music and with "Pioggia" piece at different sound pressure level.

The different effect of the musical pieces is due to the fact that "Pioggia" piece differs from the first one being inconstant in the rhythm. In particular, the rhythm is defined in an agogic accent with a rubato, therefore, it is sometimes slow and sometimes accelerated. Therefore, the complex entirety of the piece (which includes rhythm, key's definition, notes, accents, chords, etc) influenced in a significant way the enzyme activity.

A comparison with the open literature is very difficult because of only the effect of single sound frequency on the enzyme activity was deeply investigated. Besides, with a musical piece different sound frequencies stimulate the biological system in the time, on the contrary, a single frequency sound exerts the same invariant action in the time [18]. In any case, this work demonstrated as a musical piece, characterized by a peculiar rhythm (with pause and silence) and different frequencies, exerts an action (positive or negative) on a biological system.

\section{Conclusions}

In this work the effect of two musical pieces ("Reminiscenza" and "Pioggia") on the tyrosinase activity during the conversion of 1-tyrosine in 1-DOPA was investigated. The pieces were ad hoc composed giving to each one distinctive peculiarities in rhythm and range of fundamental frequencies.

A positive effect was exercised by the "Reminiscenza" piece, which determined an increase of the specific activity of about $30 \%$ than the value measured in the absence of sound. On the other hand, "Pioggia" piece had a detrimental effect on the enzymatic process. In fact, a time lag on the 1-DOPA production during the first minutes of the reaction was detected. After this period, an increase of the reaction velocity was observed even if the enzyme activity was lower than the value obtained in the absence of music.

These pioneering results highlight how musical pieces, characterized by peculiar frequencies and rhythms, can exert an action on biological elements. 
Authors wish to thank the composer G. Formigari-Bernardelli for providing his musical pieces for the experimental tests and Dr. G. M. Insana (DF Medica, Italy) for the fruitful discussion. In addition, authors wish to thank the I.E.S. Integrated Engineering Services Srl (Rende, Italy) for the sound pressure level measurements.

\section{References}

1. E.V. Rokhina, P. Lens, Trends Biotechnol. 27 (2009)

2. J.V. Sinisterra, Ultrasonics 30 (1992)

3. W.P. Barber, J. Chart, Inst. Water Environ. Manag. 19 (2005)

4. Y.M. Xiao, Q. Wu, Y. Cai, X. Lin, Carbohydr. Res. 340 (2005)

5. C. Li, M. Yoshimoto, H. Hogata, N. Tsukuda, K. Fukunaga, K. Nakao, Ultrason. Sonochem. 12 (2005)

6. W.P. Barber, J. Chart. Inst. Water Environ. Manag. 19 (2005)

7. J. Liu, Q. Wang, D. Karagić, X. Liu, J. Cui, J. Gui, M. Gu, W. Gao, Sci. Rep. 6 (2016)

8. N. Ekici, F. Dane, L. Mamedova, I. Metin, M. Huseyinov, Asian J. Plant. Sci. 62 (2007)

9. G. Shaobin, Y. Wu, K. Li, S. Li, S. Ma ,Q. Wang, R. Wang, Colloid Surface B. 78 (2010)

10. N. Saigusa, S. Imayama, Y. Teramoto, Afr. J. Biochem. Res. 9 (2015)

11. V. Chivukula, S. Ramaswamy, Plants. Int. J. Environ. Sci. Dev. 5 (2014)

12. C. Ventura, D. Gullà, M. Graves, A. Bergonzoni, R. Tassinari, J. von Stietencron, CellR4 5, 2 (2017)

13. S.E. Soh, M.E. Morris, J.L. McGinley, Parkinsonism Relat. D. 17 (2011)

14. C. Algieri, L. Donato, L. Giorno, Biotech. App. Biochem. 641 (2017)

15. L. Donato, C. Algieri, A. Rizzi, L. Giorno, J. Membrane Sci. 454 (2014)

16. D.J. Lennard, Athl. Training. 37 (2002)

17. W. Xiujuan, W. Bochu, J. Yi, L. Defang, D. Chuanren, Y. Xiaocheng, A. Sakanishi, Colloid Surface B. 27, 59 (2003)

18. B. Smagowska, M. P. Łuszczyńska, Bibliographic Review. Int. J. Occup. Saf. Erg. 19 (2013) 